\section{hommes}

\section{Hommes \& migrations}

Revue française de référence sur les dynamiques

migratoires

$1303 \mid 2013$

Diasporas marocaines

\title{
Les Marocains au Canada : histoire, profil et enjeux
}

\section{Myriam Abouzaïd et Houda Asal}

\section{(apenEdition \\ Journals}

\section{Édition électronique}

URL : http://journals.openedition.org/hommesmigrations/2560

DOI : 10.4000/hommesmigrations.2560

ISSN : 2262-3353

Éditeur

Musée national de l'histoire de l'immigration

Édition imprimée

Date de publication : 1 juillet 2013

Pagination : 85-91

ISBN : 978-2-919040-23-0

ISSN : $1142-852 X$

\section{Référence électronique}

Myriam Abouzaïd et Houda Asal, «Les Marocains au Canada : histoire, profil et enjeux », Hommes \& migrations [En ligne], 1303 | 2013, mis en ligne le 31 décembre 2015, consulté le 19 avril 2019. URL http://journals.openedition.org/hommesmigrations/2560; DOI : 10.4000/hommesmigrations.2560 


\title{
LES MAROCAINS AU CANADA HISTOIRE, PROFIL ET ENJEUX
}

par MYRIAM ABOUZAÏD, sociolinguiste, Lidilem, université de Grenoble, et HOUDA ASAL, socio-historienne, Eris-Centre Maurice-Halbwachs ${ }^{1}$

\author{
Les immigrés marocains au Canada résident dans leur grande \\ majorité dans la province francophone du Québec. Leur présence \\ y est ancienne et concernait au départ des juifs marocains. \\ Mais les migrants arrivés à partir des années 1990 subissent à \\ I'heure actuelle de sérieuses difficultés d'insertion économique \\ et sociale, à l'instar des autres migrants maghrébins. \\ Des dynamiques de regroupement voient le jour en fonction \\ des problèmes auxquels ils sont confrontés.
}

\section{L'immigration marocaine au Canada, histoire et profil}

\begin{abstract}
À la fin des années 1960, le Canada ouvre ses frontières à une immigration de plus en plus diversifiée et mène, depuis lors, une politique d'immigration permanente avec des chiffres allant de 200000 à 250000 admissions par année. Aujourd'hui, près de $20 \%$ des 33 millions d'habitants recensés au Canada sont nés à l'étranger. Depuis vingt ans, le nombre de migrants en provenance du Maghreb ne cesse d'augmenter, se dirigeant très majoritairement vers le Québec, la province qui bénéficie des plus importantes prérogatives en matière de sélection migratoire. Cela place le Maroc et l'Algérie aux deux
\end{abstract}

premiers rangs des pays d'immigration au Québec. À l'heure actuelle, la population d'origine marocaine sélève à près de 72000 personnes pour l'ensemble du Canada, dont 60000 résident au Québec. Les immigrants originaires du Maroc sont en moyenne très qualifiés et déjà francophones à leur arrivée au Québec. Cependant, ils n'échappent pas aux difficultés d'insertion socio-économique. Il s'agit donc de faire le point sur l'histoire et le profil de l'immigration marocaine au Canada et de proposer quelques pistes de réflexion sur les enjeux spécifiques auxquels se confronte cette population, 
au Québec en particulier. Avant les années 1960, les migrants originaires du monde arabe qui se dirigent vers le Canada viennent du Machrek (Syrie, Liban et Égypte, principalement). Les premières migrations en provenance du Maghreb sont plus tardives et concernent en grande majorité des Marocains de confession juive. En effet, une grande partie de la communauté juive du Maroc émigre au Québec entre 1950 et 1980, suite à l'indépendance du royaume et aux premières guerres israéloarabes $^{2}$. Cette migration se tarit par la suite, hormis quelques regroupements familiaux et des étudiants venus se former après les indépendances.

La décennie 1990 va marquer l'arrivée d'une nouvelle vague migratoire en provenance de cette région, qui ne cessera d'augmenter depuis. Il s'agit d'abord d'Algériens fuyant la guerre et bénéficiant du statut de réfugié ou de migrant économique qualifié. Ce sera ensuite le tour des Marocains qui arrivent en grand nombre à la fin des années 1990. Ces nouveaux arrivants, principalement musulmans, ont un profil et une

En provenance du Maroc, 3000 personnes entrent au Québec chaque année, ce qui représente $8 \%$ des admissions totales de la province. expérience d'intégration très différents des premiers migrants venus du Maroc, ce qui justifie qu'ils soient étudiés à part. Cette nouvelle migration marocaine se caractérise par un fort accroissement continu depuis une quinzaine d'années et par sa concentration dans la province du Québec (à 80 \%), et dans la ville de Montréal. Cette tendance explique le fait que les recherches concernant les Marocains - et les Maghrébins en général - portent toutes sur la province francophone $^{3}$. Le plus frappant réside dans l'importance de cette migration, proportionnellement à celles d'autres pays : le Maghreb à lui seul comptabilise $18 \%$ des admissions totales du Québec. En provenance du Maroc, 3000 personnes entrent au Québec chaque année, ce qui représente $8 \%$ des admissions totales de la province ${ }^{4}$.

Cette immigration massive s'explique par la coïncidence entre un contexte économique et social difficile au Maroc et la fermeture des frontières européennes, alors que la politique volontariste canadienne favorise les migrants économiques qualifiés, et celle du Québec, les francophones plus spécifiquement. Depuis les années 1970, le Québec a le droit de sélectionner les migrants indépendants qui souhaitent s'installer dans la province, alors que l'immigration familiale et humanitaire dépend entièrement du gouvernement fédéral. La grille de sélection migratoire du Québec vise à accueillir une immigration jeune, très instruite et francophone. Les critères d'admission sont, par ordre d'importance : le niveau de scolarité, les connaissances linguistiques, l'expérience de travail, lâge, le fait d'avoir un emploi réservé et l'adaptabilité. Les données statistiques montrent que les migrants marocains remplissent effectivement ces critères. Ils sont très qualifiés, puisque plus du tiers d'entre eux possède un grade universitaire (35 \%), comparativement à 16,5\% de la population québécoise en général. Ils sont également jeunes (41,6\% ont entre 25 et 44 ans, contre $27,8 \%$ pour l'ensemble de la population québécoise) et francophones (96 \% des Marocains du Québec déclarent connaître le français) $)^{5}$. Les politiques d'immigration québécoises encouragent donc certaines catégories de migrants, mais aussi certains pays de provenance, de manière plus ou moins directe. Le Québec ayant le droit 
d'avoir ses propres agents et ses bureaux d'immigration à l'étranger, en plus de ceux du gouvernement fédéral, ses choix d'implantation montrent bien les régions qu'il souhaite privilégier. Les Services d'immigration du Québec (SIQ) sont situés dans sept villes : Bruxelles, Paris, Londres, Munich, Mexico, New York et Tokyo ${ }^{6}$. En 2002, l'annonce de l'ouverture de deux bureaux dans le monde arabe semblait indiquer une orientation politique claire : "Un nouveau service d'immigration du Québec sera mis en place à Rabat, au coeur de cet important bassin d'immigration francophone qu'est le Maghreb. (...) C'est dans cette perspective de concentration des efforts sur les territoires où le français est prépondérant ou d'usage courant que s'inscrit l'ouverture de nouveaux services d'immigration au Maroc et au Liban?." Ainsi, le bassin francophone que représente le Maghreb, avec le Maroc comme centre, était clairement défini comme une région cible. Cependant, au milieu des années 2000, l'afflux de demandes commence à poser problème et les délais dans le traitement des dossiers du Maghreb sont parmi les plus longs. En 2013, les bureaux d'immigration traitent les demandes déposées fin 2010 par les candidats marocains ${ }^{8}$. En définitive, le bureau d'immigration du Québec à Rabat n’a jamais vu le jour...

\section{Dynamiques organisationnelles en diaspora}

Les dynamiques de regroupement, les expressions identitaires collectives et les mobilisations politiques que les Marocains développent au Québec dépendent de leur histoire migratoire, de leur profil et de leur expérience vécue en diaspora. Le nombre de migrants (50 000 personnes nées au Maroc pour le Québec) par rapport au nombre

de personnes d'origine marocaine (60 000 personnes déclarent une ou plusieurs origines ethniques marocaines) montre que nous sommes en présence d'une population composée majoritairement de migrants récents ${ }^{9}$.

La spécificité de la "communauté" marocaine, par rapport à l'algérienne et à la tunisienne, réside dans son histoire migratoire en deux temps et sa composition religieuse hétérogène. Les Marocains juifs étant implantés au Québec depuis longtemps, et ayant développé leurs propres réseaux et organisations, ils ne rencontrent pas les difficultés d'insertion économique et sociale des migrants arrivés à partir des années 1990. Cela a également une incidence d'un point de vue identitaire et associatif : la population juive marocaine ayant développé une identité séfarade ${ }^{10}$, elle ne se

Sous l'impulsion des autorités marocaines, le premier centre culturel marocain d'Amérique du Nord, Dar Al Maghrib, a ouvert ses portes à Montréal en 2012, avec beaucoup de retard et après avoir essuyé de nombreuses critiques de la part de la communauté qui a dénoncé un scandale financier autour de la construction de l'établissement. retrouve pas dans les dynamiques de regroupement des nouveaux migrants marocains. Il faut avoir à l'esprit les différentes appartenances identitaires qui peuvent se cumuler ou se contredire pour ces migrants récents : le facteur ethnique et/ou linguistique (marocain/arabe/berbère), l'appartenance religieuse musulmane (plus ou moins forte) et l'identification pan-régionale (maghrébine/africaine/arabe/berbère). Ainsi, analyser comment les Marocains s'organisent autour d'associations, de lieux de culte ou de fédérations unitaires permet d'évaluer la manière dont ces différentes appartenances identitaires s'expriment sur la scène publique québécoise, et montréalaise

6. Le bureau d'immigration du Québec à Damas a fermé en 2012, à cause de la guerre. Les activités ont été transférées au Québec Les demandes d'immigration des pays du Maghreb sont demeurées à Montréal, et une antenne leur est dédiée. http://www.gouv.qc.ca/ portail/quebec/international/general/delegations/7. Bulletin statistique trimestriel sur l'immigration permanente au Québec, $3^{e}$ trimestre 2004: www.micc.gouv.qc.ca/publications/pdf/BulletinStatistique 2004trimestre3 ImmigrationQuebec.pdf

8. http://www.immigration-quebec.gouv.qc.ca/fr/immigrer-installer/travailleurs-permanents/demande-immigration-general/demandeimmigration/delais-tableaux.html\#maghreb 9. Voir les tableaux sur l'origine ethnique, et les tableaux sur la citoyenneté et le pays de naissance, dans la rubrique "Immigration et diversité ethnoculturelle" : http://www12.statcan.gc.ca/nhs-enm/2011/dp-pd/dt-td/ 10. Pour la présence juive marocaine, voir notamment Yolande Cohen, "Juifs au Maroc, séfarades au Canada. Migrations et processus de construction identitaire”, op. cit.; Mikhaël Elbaz, "Ethnicité et générations en Amérique du Nord. Le cas de la seconde génération de juifs sépharades à Montréal", in Revue internationale d'action communautaire, vol. 71, n 31, 1994, pp. 63-76. 
en particulier ${ }^{11}$. Ces dernières années, pas moins d'une trentaine d'associations marocaines ont vu le jour ${ }^{12}$, mais l'enquête menée auprès de migrants maghrébins et de responsables associatifs montre que ceux-ci perçoivent la communauté (maghrébine ou, selon, marocaine ou algérienne) comme une communauté qui "manque de structure", et où "les conflits interpersonnels sont nombreux". Certains témoignages confirment que beaucoup de ces associations ont une activité limitée et parfois une durée de vie relativement courte. Les associations à vocation caritative tournées vers le pays d'origine semblent plus actives que celles qui tentent de fédérer les immigrants sur place. Il faut toutefois noter l'existence et le succès de l'École marocaine, qui propose des cours de langue et de culture (religion incluse). Créée en 1999 par un groupe de parents marocains, cette école accueille aujourd'hui 850 enfants et bénéficie, depuis 2009, du soutien financier du ministère chargé de la communauté marocaine résidant à l'étranger. Enfin, sous l'impulsion des autorités marocaines, le premier centre culturel marocain d'Amérique du Nord, Dar Al Maghrib, a ouvert ses portes à Montréal en 2012, avec beaucoup de retard et après avoir essuyé de nombreuses critiques de la part de la communauté qui a dénoncé un scandale finan-

11. Pour une analyse des dynamiques organisationnelles et des expressions identitaires des migrants originaires du Machrek, voir la thèse d'Houda Asal, “Se dire arabe au Canada. Un siècle de vie associative, entre constructions identitaires et mobilisations politiques (1882-1975)", doctorat d'histoire et civilisations, EHESS, Paris, 2011. 12. À titre d'exemples : Association des jeunes professionnels marocains (AJPM), Association Espoir-Maroc, Association marocaine de Québec (AMQ), Association des étudiants marocains au Canada (AEMC), Amicale des ressortissants marocains, Association des écoles marocaines au Canada, Association des femmes marocaines du Canada, etc. 13. Ces constats sont les résultats de l'enquête menée sur le terrain en 2011-2012 : Myriam Abouzaïd, "Langues et insertion socioéconomique : le cas de Marocains et Algériens récemment installés à Montréal”, rapport de recherche, 2013 (à paraître). 
cier autour de la construction de l'établissement ${ }^{13}$. Il faut noter que les autorités marocaines cherchent de plus en plus à maintenir un lien avec leurs ressortissants à l'étranger. Des rencontres sont ainsi organisées, via le nouveau centre culturel, à l'attention des Marocains du Québec, au regard, notamment, de la question du retour au Maroc ${ }^{14}$. Dar Al Maghrib accueille également, depuis 2013, des cours de langue amazighe (berbère), ce qui témoigne de la volonté des autorités marocaines de répercuter, au sein de la diaspora, l'évolution que le royaume connait depuis une dizaine d'années en matière de reconnaissance de sa diversité culturelle et linguistique ${ }^{15}$. Sur la question de l'expression de la berbérité des nouveaux migrants originaires du Maghreb, jusqu'à présent, la scène québécoise semble principalement occupée par des Kabyles (Berbères d'Algérie), notamment à travers le Centre Amazigh de Montréal $(\mathrm{CAM})^{16}$. Pour ce qui est des médias communautaires, il existe deux journaux maghrébins dans lesquels les Marocains jouent un rôle central : un mensuel, Maghreb Canada Express, et un bimensuel, Atlas Montréal. Ce dernier est financé par le Groupe Atlas Médias dont l'orientation est assez proche de celle du gouvernement marocain. Une étude menée en 2009 a montré qu'Atlas Montréal exprimait une identité arabo-musulmane laïque, modérée et libérale, spécifiquement maghrébine, assumant son appartenance à une société québécoise laïque, et faisant partie de la diaspora internationale maghrébine. Les thèmes principaux qu'il développe portent sur les questions de xénophobie, de racisme et d'islamophobie, sur les grands débats qui ont agité la scène publique québécoise (notamment les accommodements raisonnables), sur les questions d'immigration, de citoyenneté et d'intégration, et enfin sur les inégalités et discriminations structurelles et/ou systémiques ${ }^{17}$.

L'immigration massive et quasi concomitante en provenance des trois pays du Maghreb a, de fait, créé une expérience commune au Québec. En 2009, l'inauguration officielle du "Petit Maghreb" à Montréal a fait grand bruit. Ce quartier, anciennement italien, annonce aujourd'hui fièrement ses couleurs En 2009, l'inauguration maghrébines. Toutefois, à y regarder de plus près, il s'avère qu'il s'agit essentiellement d'un lieu de rencontre et d'un regroupement de commerçants maghrébins, et non d'un quartier résidentiel ${ }^{18}$. Si les migrants du Maghreb ne sont pas concentrés dans les mêmes quartiers, la référence à une "communauté maghrébine" semble se justifier lorsqu'il s'agit d'évoquer le profil et les difficultés officielle du "Petit Maghreb" à Montréal a fait grand bruit. Ce quartier, anciennement italien, annonce aujourd'hui fièrement ses couleurs maghrébines. Toutefois, à y regarder de plus près, il s'avère qu'il s'agit essentiellement d'un lieu de rencontre et d'un regroupement de commerçants maghrébins, et non d'un quartier résidentiel. similaires que rencontrent les Marocains, les Algériens et les Tunisiens au Québec. Ces dernières années, les nombreux débats sur l'immigration et sur les accommodements raisonnables au Québec ont largement tourné autour de l'expression des religions minoritaires dans l'espace public, et de l'islam en particulier. Plusieurs sondages d'opinion ont montré la perception de plus en plus négative des Canadiens vis-à-vis de l'islam, et des enquêtes insistent sur le fait que les médias

14. Soutenu par le gouvernement et autres instances officielles du Maroc, l'organisme Careers in Morocco a récemment mené un sondage sur “les compétences marocaines du monde et le retour au Maroc: Attentes et Réalités 2013 ". II s'agissait d'analyser les attentes et les besoins des étudiants et diplômés marocains de l'enseignement supérieur à l'étranger, et de favoriser leur réinstallation au Maroc. Voir : http://www.careersinmorocco.com/careersinmorocco/index.php 15. Myriam Abouzaïd, "Politique linguistique éducative à l'égard de l'amazighe (berbère) au Maroc : des choix sociolinguistiques et didactiques à leur mise en pratique", doctorat de sciences du langage, Lidilem, Grenoble, 2011. 16. À défaut d'une enquête qui porterait spécifiquement sur la question berbère au Québec, ces constats sont les résultats de l'enquête menée par Abouzaïd Myriam (à paraître). 17. Rachad Antonius, Marie-France René, “La diversité vue par un journal communautaire maghrébin à Montréal”, in Global Media Journal-Canadian Edition, n², 2009, pp. 91-111. 18. Manaï Bochra. "Le Petit Maghreb de Montréal, entre enjeux urbains, économiques et ethniques", communication au colloque du Criec : "Immigration, diversité ethnoculturelle et citoyenneté”, 27 janvier 2012. 19. Rachad Antonius, Noomane Raboudi, “L'islam, l’Empire et la République”, in Cahiers de recherche sociologique, $n^{\circ}$ 46, 2008 ; Maryse Potvin, "Discours sociaux et médiatiques dans le débat sur les accommodements raisonnables", in Nos diverses cités, $n^{\circ} 7,2010$, pp. 83-89; Denise Helly, "Are Muslims discriminated against in Canada since September 2001?", in Journal of Canadian Ethnic Studies, vol. 36 XXXVI, n 1, 2004, pp. 24-47. 
participent à la construction de cette image négative à la fois des Arabes et des musulmans ${ }^{19}$. Parallèlement à ces débats qui agitent la scène publique québécoise, ce sont leurs difficultés socio-économiques qui conditionnent les mobilisations politiques récentes des Marocains, et des Maghrébins plus généralement.

\section{Difficultés d'insertion économique et mobilisations politiques}

En 2008, l'annonce du taux de chômage des immigrants maghrébins amène plusieurs commentateurs à parler de "scandale" et de "honte pour le Québec" ${ }^{20}$. En effet, près d'un tiers de cette population (27,9\%), arrivée depuis
En 2009, sous l'impulsion du Marocain Monsef Derraji et de l'Algérien Lamin Foura,

le Congrès maghrébin

du Québec (CMQ) voit le jour, ayant pour objectif d'offrir des services d'orientation

en termes d'insertion professionnelle, sociale et culturelle à la communauté maghrébine. cinq ans ou moins, est en recherche d'emploi. Si tous les migrants connaissent des difficultés d'insertion professionnelle durant les cinq premières années d'installation quel que soit leur pays de départ, pour les Marocains, ces difficultés perdurent : en 2006, 18,8 \% des personnes nées au Maroc étaient au chômage, indépendamment de la durée de leur séjour au Québec, alors que la moyenne provinciale était de 7 à $8 \%$, et cela, malgré leur profil d'immigrant "idéal" décrit précédemment ${ }^{21}$.

En plus du chômage, les migrants marocains sont fréquemment confrontés au déclassement professionnel. Les statistiques indiquent que la population active expérimentée en provenance du Maroc travaille principalement dans les secteurs industriels du commerce de détail, que les hommes sont surreprésentés dans les secteurs de la fabrication, du transport et de l'entreposage, et que les femmes sont plus nombreuses à œuvrer dans le secteur des soins de santé et de l'assistance sociale ${ }^{22}$. Or, parmi les migrants marocains arrivés à partir de la fin des années 1990, nombreux sont ceux qui occupaient un emploi plus qualifié au moment du départ et possédaient un niveau de vie relativement élevé au Maroc. Il semble que la politique volontariste d'immigration québécoise ait consisté à "éveiller l'idée de migrer auprès des classes moyennes et aisées pour lesquelles la migration extérieure n'était jusqu'alors pas une nécessité économique ni une stratégie de maintien ou de promotion sociale ${ }^{23}$. Par conséquent, une grande amertume et un sentiment de tromperie et d'abandon se manifestent dans les témoignages recueillis auprès de cette population. En revanche, il apparaît que pour beaucoup, c'est la recherche d'un système de santé efficace, d'une éducation de qualité et d'un avenir meilleur pour leurs enfants qui semble les avoir amenés outre-Atlantique. Une partie de cette immigration récente peut donc être considérée davantage comme sociale que véritablement économique. Quels que soient leurs motifs d'immigration, les migrants récents venus du Maroc souffrent du chômage et de déclassement dans des proportions importantes.

Il apparait qu'en plus des difficultés habituelles rencontrées par les nouveaux arrivants au Québec (telles que la non-reconnaissance des diplômes et des expériences de travail acquises à l'étranger), les Marocains se heurtent à des obstacles spécifiques. Alors qu'ils sont déjà francophones à leur arrivée, ils ne maîtrisent pas suffisamment l'anglais, ce qui pose problème dans nombre de secteurs professionnels, surtout à Montréal. En outre, ils ne possèdent pas de réseaux professionnels et subissent des discriminations spécifiques sur le marché du travail. Sur ce dernier point, une enquête auprès d'intervenants en insertion souligne qu'on demande souvent aux migrants maghrébins (entre autres) ce qu'ils pensent du terrorisme et du 11 septembre, par exemple. Par ailleurs, les professionnels établissent également un lien étroit entre les obstacles que les 
Maghrébins plus généralement rencontrent dans leur recherche d'emploi et leurs caractéristiques "culturelles" (ils sont décrits comme passifs, rigides quant à leurs pratiques religieuses, peu à l'aise dans un rapport hiérarchique avec une femme, fermés aux interactions avec des Québécois), ce qui en dit long sur la manière dont ils sont perçus, y compris par leurs employeurs potentiels ${ }^{24}$.

En 2009, sous l'impulsion du Marocain Monsef Derraji et de l'Algérien Lamin Foura, le Congrès maghrébin du Québec (CMQ) voit le jour, ayant pour objectif d'offrir des services d'orientation en termes d'insertion professionnelle, sociale et culturelle à la communauté maghrébine. Le CMQ tente également d'exercer une force de lobbying politique. À l'occasion de son audition par l'Assemblée nationale québécoise en mai 2011, dans le cadre de la nouvelle planification de l'immigration au Québec, il a soumis un mémoire et des recommandations très concrètes pour améliorer la situation des Maghrébins au Québec (le développement de cours d'anglais, l'assouplissement des critères d'admission des ordres professionnels, la diminution des exigences en termes d'expérience de travail québécoise). Par ailleurs, le gouvernement devrait, d'une part, mener des actions de sensibilisation auprès des employeurs et de la communauté québécoise en général, pour valoriser les atouts des Maghrébins en termes de force de travail et, d'autre part, informer davantage les candidats au départ des obstacles concrets qu'ils risquent de rencontrer au Québec ${ }^{25}$.

\section{Conclusion}

La crise économique qui touche le Québec depuis quelques années, conjuguée à une conjoncture moins favorable pour les migrants en général, et certains groupes en particulier, explique les difficultés spécifiques que rencontrent les Marocains. Cette première génération de migrants marocains doit donc s'adapter à la fois à un contexte socioéconomique difficile et s'insérer dans une société québécoise qui s'interroge sur son identité et semble particulièrement préoccupée par la gestion de la pluralité religieuse. Il semblerait que "bien que les membres des groupes arabo-musulmans cherchent à participer à la vie sociale et civique canadienne et québécoise, le contexte actuel ne cesse de leur rappeler qu'ils sont des citoyens marqués par la différence ${ }^{26}$. À l'heure où une Charte de la laïcité secoue à nouveau le débat public québécois, les migrants marocains doivent parvenir à faire entendre leur voix, à la fois sur la question épineuse du chômage et des déclassements, et sur le problème plus large de la stigmatisation et des discriminations dont ils sont victimes, à l'instar d'autres minorités ${ }^{27}$.

24. Annick Lenoir-Achdjian et al., "Les difficultés d’insertion en emploi des immigrants du Maghreb au Québec : une question de perspective", op. cit. 25. http://congresmaghrebins.ca/cm/wp-content/uploads/2013/og/M\%C3\%A9moire\%20CMQ.pdf 26. Rachad Antonius, Labelle Micheline, François Rocher, Immigration, diversité et sécurité. Les associations arabo-musulmanes face à l'État au Canada et au Québec, Québec, Presses de l'université du Québec, 2009, p. 92.

27. La Charte de la laïcité est un projet du Parti québécois, publiée le 9 septembre 2013 et dont une des recommandations est l'interdiction des signes religieux pour les agents de l'État. Atlas Montréal titre "La Charte de la discorde" dans son édition du 12 au 25 septembre 2013. Plusieurs associations, dont le Congrès maghrébin au Québec, expriment leurs critiques à l'encontre d'une charte jugée "liberticide, discriminatoire et exclusive" (article publié le 11 septembre 2013: http://www.atlasmedias.com/2013/og/ a-une-charte-liberticide-discriminatoire-exclusive). Sur les chiffres des discriminations et les amalgames qui visent les musulmans, voir Denise Helly, “Les multiples visages de l'islamophobie au Canada”, in Cahiers du socialisme, n 5, 2011, pp. 99-106. 